University of Nebraska - Lincoln

DigitalCommons@University of Nebraska - Lincoln

7-20-2009

\title{
Few-cycle attosecond pulse chirp effects on asymmetries in ionized electron momentum distributions
}

\author{
Liang-You Peng \\ Department of Physics and State Key Laboratory for Mesoscopic Physics, Peking University, Beijing, \\ liangyou.peng@pku.edu.cn \\ Fang Tan \\ Department of Physics and State Key Laboratory for Mesoscopic Physics, Peking University, Beijing \\ Qihuang Gong \\ Department of Physics and State Key Laboratory for Mesoscopic Physics, Peking University, Beijing, \\ qhgong@pku.edu.cn
}

Evgeny A. Pronin

University of Nebraska-Lincoln, epronin2@unl.edu

Anthony F. Starace

University of Nebraska-Lincoln, astarace1@unl.edu

Follow this and additional works at: https://digitalcommons.unl.edu/physicsstarace

Part of the Physics Commons

Peng, Liang-You; Tan, Fang; Gong, Qihuang; Pronin, Evgeny A.; and Starace, Anthony F., "Few-cycle attosecond pulse chirp effects on asymmetries in ionized electron momentum distributions" (2009). Anthony F. Starace Publications. 169.

https://digitalcommons.unl.edu/physicsstarace/169

This Article is brought to you for free and open access by the Research Papers in Physics and Astronomy at DigitalCommons@University of Nebraska - Lincoln. It has been accepted for inclusion in Anthony F. Starace Publications by an authorized administrator of DigitalCommons@University of Nebraska - Lincoln. 


\title{
Few-cycle attosecond pulse chirp effects on asymmetries in ionized electron momentum distributions
}

\author{
Liang-You Peng, ${ }^{*}$ Fang Tan, and Qihuang Gong ${ }^{\dagger}$ \\ Department of Physics and State Key Laboratory for Mesoscopic Physics, Peking University, Beijing 100871, China
}

\author{
Evgeny A. Pronin and Anthony F. Starace \\ Department of Physics and Astronomy, The University of Nebraska, Lincoln, Nebraska 68588-0111, USA
}

(Received 1 March 2009; published 15 July 2009)

\begin{abstract}
The momentum distributions of electrons ionized from $\mathrm{H}$ atoms by chirped few-cycle attosecond pulses are investigated by numerically solving the time-dependent Schrödinger equation. The central carrier frequency of the pulse is chosen to be $25 \mathrm{eV}$, which is well above the ionization threshold. The asymmetry (or difference) in the yield of electrons ionized along and opposite to the direction of linear laser polarization is found to be very sensitive to the pulse chirp (for pulses with fixed carrier-envelope phase), both for a fixed electron energy and for the energy-integrated yield. In particular, the larger the pulse chirp, the larger the number of times the asymmetry changes sign as a function of ionized electron energy. For a fixed chirp, the ionized electron asymmetry is found to be sensitive also to the carrier-envelope phase of the few-cycle pulse.
\end{abstract}

DOI: 10.1103/PhysRevA.80.013407

Since the late 1990s both theoretical and experimental investigations of physical processes that are sensitive to the carrier-envelope phase (CEP) of few-cycle infrared (IR) laser pulses have increased significantly (see, e.g., [1-8] as well as the reviews $[9,10]$ and references therein). Over the same period, investigations of chirped (many-cycle) IR laser pulses have focused primarily on the role played by excited bound states [11-14]. A recent investigation of multiphoton ionization of the Cs atom by chirped two-cycle laser pulses (having frequency $\omega=1.55 \mathrm{eV}$ and $5.3 \mathrm{fs}$ duration) found significant effects of both the CEP and the chirp of the laser pulse on the angular distributions of photoelectrons with an energy of $1.8 \mathrm{eV}$; asymmetries were found in the yield of electrons ionized in the upper and lower hemispheres along the laser polarization axis [15]. Note that in this study, the Cs $6 p$ state is very close to the one-photon resonance [15]. Also, an earlier investigation [14] of multiphoton ionization of the $\mathrm{Na}$ atom using chirped five-cycle 13.3 fs laser pulses found no chirp effects on ionized electron angular distributions when "calculations without excited bound states" were carried out; it also found no chirp effects in calculations using a photon energy of $\omega=6.2 \mathrm{eV}$ (which exceeds the Na ionization potential of $5.14 \mathrm{eV}$ ). Thus, an open question remains concerning the effects of chirped few-cycle laser pulses when the photon energy exceeds the ionization threshold of an atom.

In the attosecond regime, few-cycle [16] and single-cycle $[17,18]$ attosecond pulses with stable and even tunable CEPs have been achieved experimentally. Significant effects of the CEP on ionized electron momentum and energy distributions have been investigated theoretically in some detail and found to be significant at intensities just above current experimental capabilities $[19,20]$. It is well known that current techniques

\footnotetext{
*liangyou.peng@pku.edu.cn

†qhgong@pku.edu.cn

†astarace1@unl.edu
}

PACS number(s): 32.80.Fb, 32.80.Rm, 32.80.Qk

for generating attosecond pulses introduce a chirp [21,22]. Recently, a theoretical investigation of the photoelectron spectrum of a coherently populated superposition of electronic states for the hydrogen atom that was ionized by a chirped many-cycle attosecond pulse was carried out [22]. Significant asymmetries were found in the photoelectron angular distributions; moreover, these asymmetries were quite sensitive to the chirp of the attosecond pulse [22].

In this paper we investigate asymmetries of ionized electron momentum distributions produced by chirped few-cycle attosecond pulses with various fixed CEPs. Our results are based on solutions of the three-dimensional time-dependent Schrödinger equation (TDSE) for the ground state of the hydrogen atom interacting with a linearly polarized chirped few-cycle attosecond pulse. In contrast to Ref. [22], we investigate here the effects of the CEPs of few-cycle chirped attosecond pulses. In contrast to Ref. [15], we investigate here the attosecond regime for pulses having photon energies that greatly exceed the atomic binding energy, and which, in particular, are not resonant with excited atomic bound states.

We consider a chirped attosecond laser pulse having a Gaussian form similar to that used by others $[14,15,22]$. The laser pulse is assumed to be linearly polarized along the $z$ axis with its vector potential given by

$$
\mathbf{A}(t)=A F(t) \sin \left[\omega(t) t+\phi_{0}\right] \mathbf{e}_{z},
$$

where $\phi_{0}$ is the CEP of the pulse, and the peak amplitude $A$, chirped carrier frequency $\omega(t)$, and time envelope $F(t)$ are given, respectively, by

$$
\begin{gathered}
A=\frac{E}{\omega_{0}}=\frac{1}{\omega_{0}} \sqrt{\frac{I_{0} / I_{\mathrm{au}}}{\sqrt{1+\xi^{2}}}}, \\
\omega(t)=\omega_{0}+4 \ln 2 \frac{\xi}{1+\xi^{2}} \frac{t}{\tau_{0}^{2}},
\end{gathered}
$$




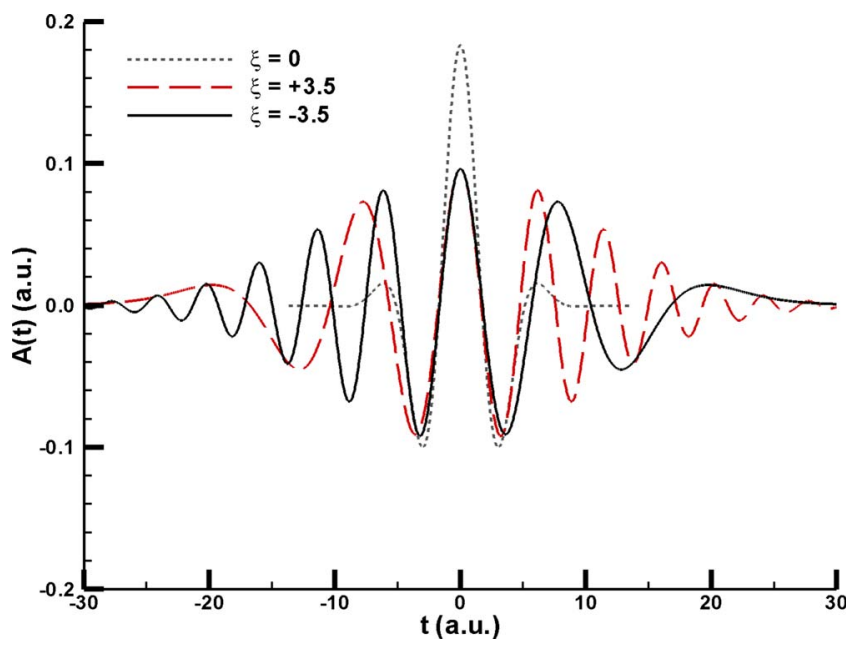

FIG. 1. (Color online) Dependence of laser-pulse vector potentials on the chirp rate $\xi$ for three cases: $\xi=0$, \pm 3.5 . Each laser pulse has a central carrier frequency $\omega_{0}=25 \mathrm{eV}, \mathrm{CEP} \phi_{0}=0.5 \pi$, intensity $I=I_{0} / \sqrt{1+\xi^{2}}$, and duration $\tau=\tau_{0} \sqrt{1+\xi^{2}}$, where $I_{0}=1$ $\times 10^{15} \mathrm{~W} / \mathrm{cm}^{2}$ and $\tau_{0}=T_{0}$.

$$
F(t)=\exp \left[-4 \ln 2 \frac{1}{1+\xi^{2}} \frac{t^{2}}{\tau_{0}^{2}}\right],
$$

in which $I_{\mathrm{au}}=3.51 \times 10^{16} \mathrm{~W} / \mathrm{cm}^{2}$ is the atomic unit of intensity and the parameter $\xi$ is the chirp rate. The case $\xi=0$ corresponds to a transform-limited pulse with carrier frequency $\omega_{0}$, peak intensity $I_{0}$, and pulse duration $\tau_{0}$ (full width at half maximum). For a chirped pulse, the peak intensity and pulse duration are given, respectively, by $I$ $=I_{0} / \sqrt{1+\xi^{2}}$ and $\tau=\tau_{0} \sqrt{1+\xi^{2}}$. The optical period corresponding to the central carrier frequency $\omega_{0}$ is defined as $T_{0}$ $=2 \pi / \omega_{0}$.

Vector potential (1) simulates a realistic experimental case in which a nonzero chirp $\xi$ leads to an increase in the pulse duration and a decrease in the pulse intensity for a fixed total pulse energy, i.e., the same pulse energy as for an unchirped pulse. Most importantly, the frequency bandwidth of the chirped pulse remains the same as that of the corresponding unchirped pulse. As examples, we compare in Fig. 1 the vector potential for an unchirped pulse with those having a positive and a negative chirp.

For a hydrogen atom interacting with a chirped laser pulse, the time-dependent Schrödinger equation is

$$
i \frac{\partial}{\partial t} \Psi(\mathbf{r}, t)=\left[-\frac{1}{2} \nabla^{2}+\frac{1}{r}-i \mathbf{A}(t) \cdot \nabla\right] \Psi(\mathbf{r}, t),
$$

which is solved numerically in spherical coordinates. Details of our numerical methods can be found in our previous works $[19,20,23]$. In brief, the wave function is expanded in spherical harmonics and the corresponding radial-wave functions are calculated using the finite difference method. The total wave function is propagated in time using the Arnoldi method. The maximum radial grid point is taken to be 2000 a.u. (with a grid spacing of $\Delta r=0.1$ a.u.) so that even the fastest electronic wave packets are not reflected at the box edge prior to the end of the pulse. In the velocity gauge description of the interaction Hamiltonian, we obtain fully converged results when the maximum angular-momentum quantum number is taken to be eight. All other parameters (such as the propagation time step and the order of the Arnoldi propagator) are carefully chosen so that all results are fully converged.

The probability that the ionized electron has momentum $\mathbf{k}$ is obtained by projecting the final wave function at time $t_{f}$ (i.e., after interaction with the laser pulse) onto the corresponding field-free continuum momentum state, $\Psi_{\mathbf{k}}^{-}(\mathbf{r})$, i.e.,

$$
P(\mathbf{k})=P(k, \theta, \varphi)=\left|\left\langle\Psi_{\mathbf{k}}^{-}(\mathbf{r}) \mid \Psi\left(\mathbf{r}, t_{f}\right)\right\rangle\right|^{2} .
$$

As $P(\mathbf{k})$ has azimuthal symmetry for a linearly polarized pulse, the $\varphi$-integrated differential ionization probability is

$$
P(E, \theta)=2 \pi \sqrt{2 E} P(k, \theta, \varphi=0),
$$

where $k=\sqrt{2 E}$, and the total ionization probability is thus

$$
P_{\text {total }}=\int_{0}^{\infty} d E \int_{0}^{\pi} \sin \theta d \theta P(E, \theta) .
$$

To quantify the asymmetry in the ionized electron momentum distributions, we define two normalized asymmetry parameters: $A_{1}$ measures the asymmetry of electrons ejected at $0^{\circ}$ and $180^{\circ}$ with respect to the laser polarization axis; $A_{2}$ is similarly defined but is calculated by integrating over a $10^{\circ}$ cone around $0^{\circ}$ and $180^{\circ}$. That is,

$$
A_{1}=\frac{P_{0}^{+}-P_{0}^{-}}{P_{0}^{+}+P_{0}^{-}},
$$

where $P_{0}^{+}=\int_{0}^{\infty} d E P(E, 0)$ and $P_{0}^{-}=\int_{0}^{\infty} d E P(E, \pi)$, and

$$
A_{2}=\frac{P_{10^{\circ}}^{+}-P_{10^{\circ}}^{-}}{P_{10^{\circ}}^{+}+P_{10^{\circ}}^{-}},
$$

where $P_{10^{\circ}}^{+}=\int_{0}^{\infty} d E \int_{0}^{10^{\circ}} \sin \theta d \theta P(E, \theta)$ and $P_{10^{\circ}}^{-}$ $=\int_{0}^{\infty} d E \int_{170^{\circ}}^{180^{\circ}} \sin \theta d \theta P(E, \theta)$. Note that $A_{1}$ and $A_{2}$ are dependent on both the chirp rate $\xi$ and the CEP $\phi_{0}$.

In this work, the transform-limited laser pulses are chosen to have a carrier frequency $\omega_{0}=25 \mathrm{eV}$, pulse duration $\tau_{0}=T_{0}$, and two peak intensities: $I_{0}=1 \times 10^{14}$ or $1 \times 10^{15} \mathrm{~W} / \mathrm{cm}^{2}$. These laser parameters have been chosen to be similar to those used in our previous studies $[19,20]$ of CEP effects for unchirped pulses in order to facilitate comparisons. Note in particular that in Ref. [20] the asymmetry parameter $A_{1}$ was shown to scale as $I_{0}^{1 / 2}$. In Fig. 2, we show the differential probability distributions for ionized electrons $P(E, \theta)$ as a function of energy $E$ along $\theta=0$ and $\theta=\pi$ for five different chirp rates $\xi$ [24]. [The values of $\xi$ are approximately those of some of the maxima of $\left|A_{1}\right|$ for the two values of the CEP in Fig. 4(c).] To see the asymmetries more clearly, we show the results at the higher intensity, $I_{0}=1$ $\times 10^{15} \mathrm{~W} / \mathrm{cm}^{2}$, for two CEPs: $\phi_{0}=0.5 \pi$ and 0 . For each case in Fig. 2, the electron distributions extend over the same energy range, as expected (since the bandwidth of a chirped laser pulse remains the same as that of a transform-limited pulse). For the case $\phi_{0}=0.5 \pi$, when $\xi=0$ [cf. Fig. 2(a)], the $\theta=0$ and $\theta=\pi$ curves have similar maximum values and become equal for an ionized electron energy of $8 \mathrm{eV}$. As $\xi$ 




FIG. 2. (Color online) Differential probabilities $P\left(E, \theta_{k}\right)$ of electrons ionized along $\theta=0$ (solid lines) and $\theta=\pi$ (dashed lines) for two CEPs, $\phi_{0}=0.5 \pi$ (left column) and $\phi_{0}=0$ (right column), and five chirp rates $\xi$ (indicated in each panel). The laser pulse for $\xi=0$ has central carrier frequency $\omega_{0}=25 \mathrm{eV}$, intensity $I_{0}=1$ $\times 10^{15} \mathrm{~W} / \mathrm{cm}^{2}$, CEP $\phi_{0}=0.5 \pi$, and duration $\tau_{0}=T_{0}$.

increases from 0 to 1.5 [cf. Fig. 2(b)], the energy at which the curves intersect shifts from 8 to $17 \mathrm{eV}$, and the curve for $\theta=0$ has a slightly higher peak value than that for $\theta=\pi$. In addition, a new intersection point occurs near $27 \mathrm{eV}$. When the sign of the chirp is reversed [cf. Fig. 2(c)], the phenomena are very similar, with the intersection points shifting only slightly compared to those for positive chirp.

For a CEP $\phi_{0}=0$, the asymmetry is barely observable for $\xi=0$. However, as one increases $\xi$ from 0 to 0.75 [cf. Fig. $2(\mathrm{e})]$, the asymmetry between electrons ionized along $\theta=0$ and $\pi$ is significantly increased. As in the case of $\phi_{0}=0.5 \pi$, there are two intersection points, in this case near 4 and 23.5 $\mathrm{eV}$. However, the most significant difference for the case of $\phi_{0}=0$ is that the two curves along $\theta=0$ and $\pi$ exchange their relative magnitude when one reverses the sign of the chirp rate $\xi$ [cf. Figs. 2(e) and 2(f)]. In other words, for $\phi_{0}=0$ the asymmetry factor $A_{1}$ changes its sign when one reverses the sign of the chirp rate. For a CEP $\phi_{0}=0.5 \pi$, however, the asymmetry factor $A_{1}$ does not change its sign when one reverses the sign of the chirp rate.

In order to see clearly the intersection points and their energy positions as $\xi$ is changed, it is useful to evaluate the difference between the differential probabilities along $\theta=0$ and $\pi$ as a function of the electron energy, i.e.,

$$
D(E)=P(E, 0)-P(E, \pi) .
$$

In Fig. 3, we plot $D(E)$ for two laser intensities $I_{0}=1$ $\times 10^{14}$ and $1 \times 10^{15} \mathrm{~W} / \mathrm{cm}^{2}$, two CEPs $\phi_{0}=0$ and $0.5 \pi$, and four chirp rates $\xi$ indicated in each panel. The most striking feature is that, for both laser intensities, the curves of $D(E)$ for the same CEP $\phi_{0}$ show the same structure but have higher magnitudes for higher intensity. When $D(E)=0$, the probabilities for electrons ejected along $\theta=0$ and $\pi$ are equal. For $I_{0}=1 \times 10^{14} \mathrm{~W} / \mathrm{cm}^{2}$ and $\xi=0, D(E)$ equals zero only once [cf. Fig. 3(a)]. However as $\xi$ increases to $0.75,1.5$,

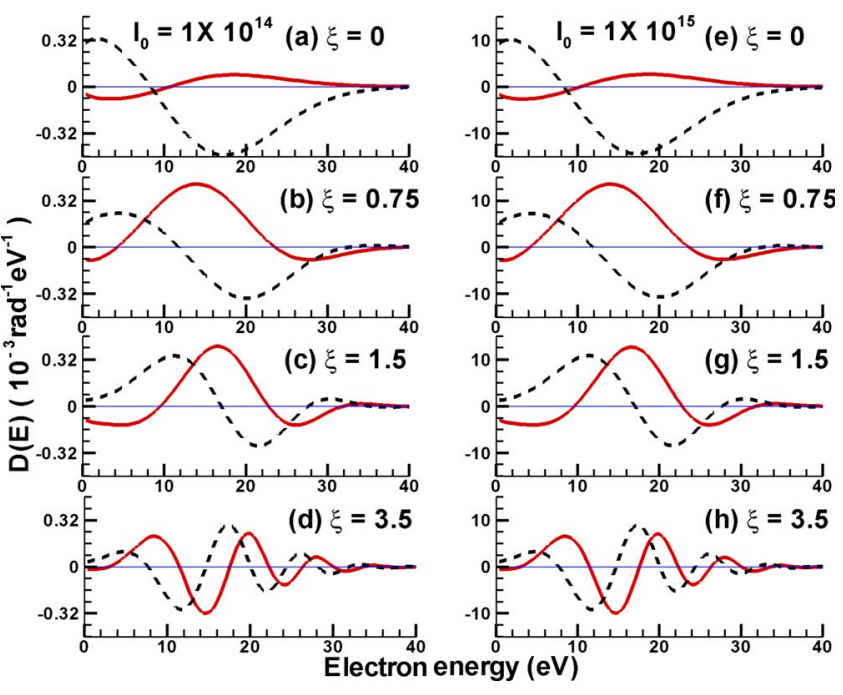

FIG. 3. (Color online) Differential probabilities, $D(E)$ [cf. Eq. (11)], for two CEPs, $\phi_{0}=0$ (solid lines) and $\phi_{0}=0.5 \pi$ (dashed lines), two laser intensities, $I_{0}=1 \times 10^{14}$ (left column) and $I_{0}=1$ $\times 10^{15} \mathrm{~W} / \mathrm{cm}^{2}$ (right column), and four chirp rates $\xi$ shown in each panel. Other laser parameters are the same as in Fig. 2.

and 3.5, respectively, in Figs. 3(b)-3(d), the number of zeros increases to two, three, and eight, respectively, over the energy range shown. Thus, the number of oscillations of $D(E)$ increases as $\xi$ increases. Moreover, as shown in Fig. 3(d), the oscillation period in energy decreases as the electron energy increases. Most significantly, whereas for $\xi=0$, the asymmetry for a pulse having a CEP $\phi_{0}=0$ is far smaller than that for one having a CEP $\phi_{0}=0.5 \pi$; for a pulse with nonzero chirp, the asymmetries for the two CEPs become of comparable magnitude (although their maxima occur at different energies).

In Fig. 4, we plot the asymmetry parameters $A_{1}$ and $A_{2}$ as continuous functions of $\xi$ (over the range $-3.5 \leq \xi \leq+3.5$ ) for two laser intensities $I_{0}=1 \times 10^{14}$ (left column) and 1 $\times 10^{15} \mathrm{~W} / \mathrm{cm}^{2}$ (right column), and two CEPs: $\phi_{0}=0$ (solid curves) and $0.5 \pi$ (dashed curves). For the same CEPs $\phi_{0}$, the asymmetry parameters $A_{1}$ for both laser intensities show very similar oscillatory structures. In fact, results for the two intensities overlap each other exactly when we divide the higher intensity curve by a factor of 3.138 , which is close to the ratio of the peak electric field strengths (i.e., 3.16). This is consistent with the intensity scaling law shown in Fig. 3 of Ref. [20], i.e., in which the asymmetry parameters scale with the square root of the laser intensity. Note also that $A_{2}$, which is integrated over a $10^{\circ}$ cone, shows very similar structure to $A_{1}$ but may be easier to measure experimentally.

The oscillatory structure of $A_{1}$ as a function of the chirp rate is caused by the chirp itself, and not by the increase in the laser-pulse length with increasing chirp (i.e., $\tau=\tau_{0} \sqrt{1+\xi^{2}}$ ). This has been confirmed by calculations with transform-limited pulses having the same peak laser intensity, $I=I_{0} / \sqrt{1+\xi^{2}}$, and the same pulse duration $\tau$ as the corresponding chirped pulse. As expected, the asymmetry factor $A_{1}$ for these transform-limited pulses decreases uniformly with increase in the pulse length and does not show any oscillations as a function of the pulse length. 

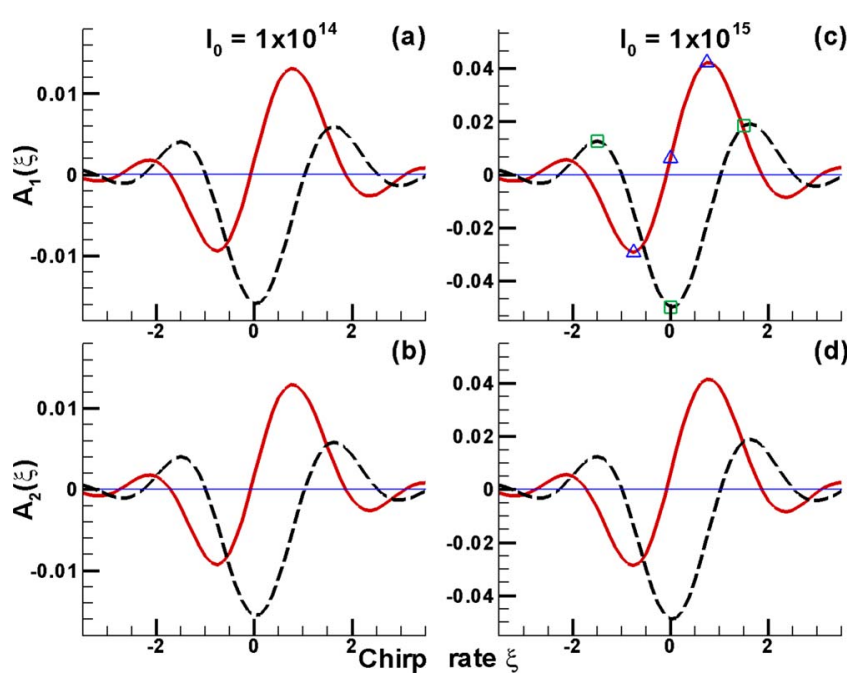

FIG. 4. (Color online) Comparison of the asymmetry parameters $A_{1}$ (first row) and $A_{2}$ (second row) [cf. Eqs. (9) and (10)] vs chirp rate $\xi$ for two laser intensities: $I_{0}=1 \times 10^{14}$ (left column) and 1 $\times 10^{15} \mathrm{~W} / \mathrm{cm}^{2}$ (right column). In each panel, results for two CEPs are given: $\phi_{0}=0$ (solid curve) and $\phi_{0}=0.5 \pi$ (dashed curve). Panel (c) also gives $P(E, \theta)$ results from Fig. 2 for $\phi_{0}=0$ (triangles) and $\phi_{0}=0.5 \pi$ (squares).

As shown in Fig. 4, the behaviors of the asymmetry parameters for the two values of the CEP differ markedly. For $\phi_{0}=0, A_{1}(\xi)$ vanishes at a small negative chirp. As the magnitude of the chirp increases from zero, the asymmetry parameters quickly rise (decrease) to their maximum (minimum) values for positive (negative) chirps in the range 0.5 $\leq|\xi| \leq 0.75$. For larger chirp magnitudes $|\xi|$, the asymmetry parameters oscillate with decreasing amplitudes. For $\phi_{0}$ $=0.5 \pi$, on the other hand, the asymmetry parameters take their maximum absolute values at a small positive chirp. For larger chirp magnitudes $|\xi|$, these asymmetry parameters oscillate with decreasing amplitudes. Finally, careful examination of Fig. 4 shows that the curves for $\phi_{0}=0\left(\phi_{0}=0.5 \pi\right)$ are only qualitatively antisymmetric (symmetric) with respect to positive and negative chirps of the same magnitudes. Thus, the ionized electron asymmetries for positive and negative chirps of equal magnitude differ in this case in which the photon energy is well above the ionization threshold.

In Fig. 5, we show $A_{1}$ as a function of $\phi_{0}$ for (a) $\xi=0$, \pm 0.75 , and (b) $\xi=0, \pm 1.5$ for a few-cycle attosecond pulse of intensity $I_{0}=1 \times 10^{15} \mathrm{~W} / \mathrm{cm}^{2}$. The asymmetry parameter $A_{2}$ is very similar to $A_{1}$ and is thus not shown. One sees from Fig. 5 that the curve for $\xi=0$ is approximately sinusoidal in the CEP. As the chirp rate $\xi$ is increased to 0.75 in Fig. 5(a), the curve approximates a cosine function and the maximum value of $A_{1}$ decreases slightly. Interestingly, when the sign of $\xi$ is reversed, i.e., $\xi=-0.75$, the corresponding asymmetry parameter not only changes sign but is also phase shifted, indicating another difference between positive and negative chirp rates. As $\xi$ is further increased to 1.5 in Fig. 5(b), the changes are similar but more extreme: the amplitudes of oscillation decrease greatly and the phase shift between the curves for positive and negative chirps is much greater.

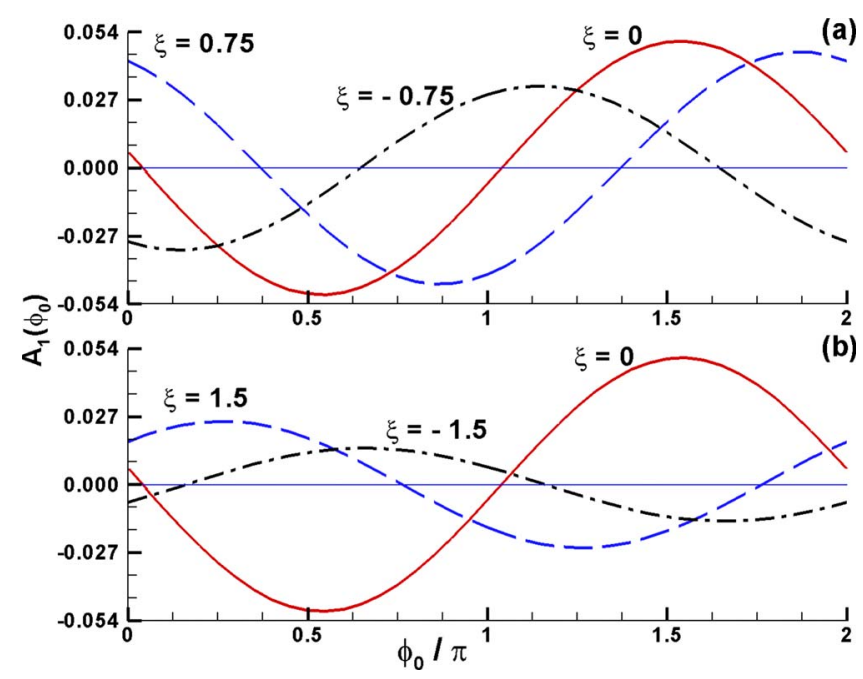

FIG. 5. (Color online) Dependence of the asymmetry parameter $A_{1}$ on the CEP $\phi_{0}$ for attosecond pulses with $I_{0}=1 \times 10^{15} \mathrm{~W} / \mathrm{cm}^{2}$ and five chirp values, indicated above each curve.

Before concluding, some remarks on the interpretation of these results are in order. First, the finding in Ref. [20] that the asymmetry parameter $A_{1}$ in Eq. (9) scales with intensity as $I^{1 / 2}$ was attributed to an interference between transition amplitudes of first and second orders in the laser pulse. This is consistent with interpretations of similar asymmetries found in photoexcited currents in semiconductors [25] as well as in ionization of Rydberg atoms by few-cycle radiofrequency pulses [26]. It is also consistent with the general framework of Roudnev and Esry [27] for understanding CEP effects in quantum systems interacting with an intense short laser pulse, in which such effects are attributed to interference between transition amplitudes involving odd and even numbers of photons. All of these analyses were for the case of transform-limited pulses. In contrast to an unchirped pulse, which has a fixed carrier frequency and a fixed CEP, a chirped pulse has a time-varying carrier frequency or, from an alternative point of view, has a time-varying effective CEP: namely, the fixed CEP plus the time-varying part of the carrier frequency. Considered as a function of time, the asymmetry in the ionized electron distribution produced by a chirped pulse is thus much more sensitive to the time at which the electron is ionized. From an energy point of view, the asymmetry becomes a much more sensitive function of the ionized electron energy. Our results accurately portray this sensitivity.

Second, we have carried out strong-field approximation (SFA) calculations for few-cycle chirped pulses with central carrier frequency $\omega_{0}=25 \mathrm{eV}$ in order to confirm that our present TDSE results are not sensitive to Rydberg levels in the $\mathrm{H}$ atom. In these SFA calculations, the initial state was the $\mathrm{H}$ atom ground state and the final states were Volkov states. The ionized electron asymmetries were found to be qualitatively similar to the exact results presented here.

In summary, in this paper we have analyzed numerically the asymmetries of ionized electron momentum distributions produced by chirped few-cycle attosecond pulses having various fixed CEPs. The central carrier frequency of the 
pulse is chosen to be $25 \mathrm{eV}$, which is well above the ionization threshold, so that the contribution of excited states is negligible, thus allowing us to focus on the effects of the pulse chirp. Our results are based on solutions of the threedimensional time-dependent Schrödinger equation for the ground state of the hydrogen atom interacting with a linearly polarized chirped few-cycle attosecond pulse. Our results allow one to make the following conclusions: first, for fewcycle attosecond pulses having even a small chirp, the asymmetry in the ionized electron momentum distribution can be changed significantly and is sensitive to the sign of the chirp. Second, this asymmetry is also quite sensitive to the CEP of the pulse, even for chirped pulses; the maximum asymmetry is very sensitive to both the chirp and the CEP, occurring for nonzero but small values of the chirp. Third, regarding the energy distributions along $\theta=0$ and $\theta=\pi$ for chirped pulses, the asymmetry can vanish at particular electron energies that are very sensitive to the chirp. Finally, the present results demonstrate clearly that asymmetries in the momentum distributions of electrons ionized by few-cycle chirped attosecond pulses are highly sensitive to both the CEP and the chirp of the pulse, and, consequently, may prove useful to experimentalists for characterizing their pulses.

We thank Cornelis J. Uiterwaal for detailed discussions concerning chirped pulses. This work is supported in part by National Natural Science Foundation of China Grants No. 10704003 and No. 10821062, National Basic Research Program of China Grant No. 2006CB806007, and U.S. Department of Energy Grant No. DE-FG02-96ER14646.
[1] E. Cormier and P. Lambropoulos, Eur. Phys. J. D 2, 15 (1998).

[2] A. de Bohan, P. Antoine, D. B. Milošević, and B. Piraux, Phys. Rev. Lett. 81, 1837 (1998).

[3] I. P. Christov, Opt. Lett. 24, 1425 (1999).

[4] G. G. Paulus, F. Grasbon, H. Walther, P. Villorese, M. Nisoli, S. Stagira, E. Priori, and S. De Silvestri, Nature (London) 414, 182 (2001).

[5] D. B. Milošević, G. G. Paulus, and W. Becker, Phys. Rev. Lett. 89, 153001 (2002).

[6] G. G. Paulus, F. Lindner, H. Walther, A. Baltuška, E. Goulielmakis, M. Lezius, and F. Krausz, Phys. Rev. Lett. 91, 253004 (2003).

[7] T. Nakajima and S. Watanabe, Phys. Rev. Lett. 96, 213001 (2006); Opt. Lett. 31, 1920 (2006).

[8] A. J. Verhoef, A. Fernández, M. Lezius, K. O’Keefe, M. Uiberacker, and F. Krausz, Opt. Lett. 31, 3520 (2006).

[9] T. Brabec and F. Krausz, Rev. Mod. Phys. 72, 545 (2000).

[10] D. B. Milošević, G. G. Paulus, D. Bauer, and W. Becker, J. Phys. B 39, R203 (2006).

[11] R. Marani and E. J. Robinson, J. Phys. B 32, 711 (1999).

[12] B. Chatel, J. Degert, S. Stock, and B. Girard, Phys. Rev. A 68 , 041402(R) (2003).

[13] M. Wollenhaupt, A. Präkelt, C. Sarpe-Tudoran, D. Liese, and T. Baumert, Appl. Phys. B: Lasers Opt. 82, 183 (2006).

[14] T. Nakajima, Phys. Rev. A 75, 053409 (2007).

[15] T. Nakajima and E. Cormier, Opt. Lett. 32, 2879 (2007).

[16] A. Baltuška, Th. Udem, M. Uiberacker, M. Hentschel, E. Goulielmakis, Ch. Gohle, R. Holzwarth, V. S. Yakovlev, A. Scrinzi, T. W. Hänsch, and F. Krausz, Nature (London) 421,
611 (2003).

[17] G. Sansone, E. Benedetti, F. Calegari, C. Vozzi, L. Avaldi, R. Flammini, L. Poletto, P. Villoresi, C. Altucci, R. Velotta, S. Stagira, S. De Silverstri, and M. Nisoli, Science 314, 443 (2006).

[18] E. Goulielmakis, M. Schultze, M. Hofstetter, V. S. Yakovlev, J. Gagnon, M. Uiberacker, A. L. Aquila, E. M. Gullikson, D. T. Atwood, R. Kienberger, F. Krausz, and U. Kleineberg, Science 320, 1614 (2008).

[19] L. Y. Peng and A. F. Starace, Phys. Rev. A 76, 043401 (2007).

[20] L. Y. Peng, E. A. Pronin, and A. F. Starace, New J. Phys. 10, 025030 (2008).

[21] P. Agostini and L. F. DiMauro, Rep. Prog. Phys. 67, 813 (2004).

[22] G. L. Yudin, A. D. Bandrauk, and P. B. Corkum, Phys. Rev. Lett. 96, 063002 (2006).

[23] L. Y. Peng and A. F. Starace, J. Chem. Phys. 125, 154311 (2006).

[24] Note that in Ref. [20], we plotted the electron distribution differential in momentum along the laser polarization axis, with the distributions plotted vs electron energy. Here, we plot the electron distribution differential in energy along the laser polarization axis.

[25] E. Dupont, P. B. Corkum, H. C. Liu, M. Buchanan, and Z. R. Wasilewski, Phys. Rev. Lett. 74, 3596 (1995).

[26] A. Gürtler, F. Robicheaux, W. J. van der Zande, and L. D. Noordam, Phys. Rev. Lett. 92, 033002 (2004).

[27] V. Roudnev and B. D. Esry, Phys. Rev. Lett. 99, 220406 (2007). 